\title{
NOTE ON ASYMPTOTIC EXPRESSIONS IN THE THEORY OF LINEAR DIFFERENTIAL EQUATIONS.*
}

BY PROFESSOR W. E. MILNE.

(Read before the American Mathematical Society, December 28, 1915.) tion

LET $n$ independent solutions of the linear differential equa-

$$
\frac{d^{n} u}{d x^{n}}+P_{2}(x) \frac{d^{n-2} u}{d x^{n-2}}+\cdots+P_{n}(x) u+\rho^{n} u=0
$$

be denoted by $y_{1}, y_{2}, \cdots, y_{n}$. It is the aim of this note to establish asymptotic representations of a particular form for the $n$ functions $\bar{y}_{1}, \bar{y}_{2}, \cdots, \bar{y}_{n}$ determined by the $n$ identities

$$
\sum_{i=1}^{n} y_{i}{ }^{(s-1)} \bar{y}_{i}=\left\{\begin{array}{l}
0, \text { if } s=1,2, \cdots, n-1, \\
1, \text { if } s=n .
\end{array}\right.
$$

For this purpose we employ asymptotic forms for the $y$ 's, as follows. $t$ If the coefficients $P_{s}(x)$ in (1) have continuous derivatives of order $(m+n-s), m$ being a positive integer or zero, in the interval $a \leqq x \leqq b$, then there exist $n$ independent solutions of (1) of the form

where

$$
\begin{aligned}
y_{i} & =u_{i}(x, \rho)+e^{\rho w_{i}(x-c)} E_{i 0} / \rho^{m+1}, \\
y_{i}{ }^{(k)}= & u_{i}{ }^{(k)}(x, \rho)+e^{\rho w_{i}(x-c)} E_{i k} / \rho^{m+1-k} \\
& \quad(i=1,2, \cdots, n ; k=1,2, \cdots, n-1),
\end{aligned}
$$

$$
u_{i}(x, \rho)=e^{\rho w_{i}(x-c)}\left[1+\frac{\varphi_{1}(x)}{\rho w_{i}}+\cdots+\frac{\varphi_{m}(x)}{\left(\rho w_{i}\right)^{m}}\right] .
$$

The functions $\varphi_{j}(x)$ have continuous $(m+n-j)$ th derivatives in $(a, b)$ and are independent of $i$, while for $x$ in $(a, b)$

* The formulas given here were published without proof in the Proceedings Nat. Academy of Sciences, vol. 2 (1916), pp. 543-5.

$\dagger$ The existence of asymptotic solutions of $(1)$ in nearly the form given in (3) was proved by Birkhoff, Transactions Amer. Math. Society, vol. 9 (1908), pp. 219-231, 381-2. The proof of the formulas in (3) and (4) is conducted in a similar manner and offers no essentially new difficulty. For an explicit statement of the difference between Birkhoff's formulas and those here given, see the note in the Proceedings referred to above. 
and $\rho$ in a suitably chosen sector* $S_{k}$ of the $\rho$-plane the $E_{i j}$ are analytic in $\rho$ and bounded as $\rho$ becomes infinite.

By the use of the above formulas we can now show that when the coefficients $P_{s}(x)$ have continuous $(m+n-s)$ th derivatives in $(a, b)$, the $n$ functions $\bar{y}_{i}$ have when $|\rho|$ is large the asymptotic form $\dagger$

$$
\begin{aligned}
\bar{y}_{i}=\frac{e^{-\rho w_{i}(x-c)}}{n\left(\rho w_{i}\right)^{n-1}}\left[\bar{v}_{i}(x, \rho)+\bar{E}_{i} / \rho^{m+1}\right] & \\
& (i=1,2, \cdots, n),
\end{aligned}
$$

where

$$
\bar{v}_{i}(x, \rho)=1+\psi_{1}(x) / \rho w_{i}+\cdots+\psi_{m}(x) /\left(\rho w_{i}\right)^{m} .
$$

The functions $\psi_{j}(x)$ are independent of $i$ and have continuous $(m+n-j)$ th derivatives in $(a, b)$, and for $x$ in $(a, b)$ and $\rho$ on $S_{k}$ the $\bar{E}_{i}$ are analytic in $\rho$ and bounded as $\rho$ becomes infinite.

The method of proof is to substitute in (2) from (5) and (6) and show that the $m \psi$ 's and $n \bar{E}$ 's can be chosen to satisfy (2). It will readily be seen that $y_{i}{ }^{\left({ }^{k}\right)}$ can be written

where

$$
\begin{aligned}
y_{i}{ }^{(k)}=\left(\rho w_{i}\right)^{k} e^{\rho w_{i}(x-c)}\left[v_{i k}(x, \rho)+E_{i k}{ }^{\prime} / \rho^{m+1}\right], \\
(i=1,2, \cdots, n ; k=0,1, \cdots, n-1),
\end{aligned}
$$

$$
v_{i k}(x, \rho)=1+\varphi_{1 k} / \rho w_{i}+\cdots+\varphi_{m k} /\left(\rho w_{i}\right)^{m},
$$

and

$$
\varphi_{j k}=\sum_{r+t=j}\left[\frac{k !}{r !(k-r) !}\right] \varphi_{t}^{(r)}
$$

Substitute in (2) from (5) and (7), cancel the exponentials, and divide out the factor $-1 / n \rho^{n-s}$ from the $s$ th equation. The result is $\ddagger$

$$
\begin{aligned}
& \sum_{i=1}^{n} w_{i}{ }^{s} v_{i, s-1} \bar{v}_{i}+\frac{1}{\rho^{m+1}}[\left.\sum_{i=1}^{n}\left(w_{i}{ }^{s}+E / \rho\right) \bar{E}_{\imath}+E\right] \\
&=\left\{\begin{array}{r}
0, \text { if } s=1,2, \cdots, n-1, \\
-n, \text { if } s=n .
\end{array}\right.
\end{aligned}
$$

* For the precise definition of $S_{k}$ see either Birkhoff, loc. cit., or the note in the Proceedings.

$\dagger$ A similar but less explicit formula was used by Birkhoff, loc. cit., p. 391 , formula (56).

$\ddagger$ The letter $E$ is here used in a generic sense to denote any function analytic in $S_{k}$ and bounded in $S_{k}$ as $\rho$ becomes infinite. 
By (6) and (8)

$$
\sum_{i=1}^{n} w_{i}{ }^{s} \eta_{i, s-1} \bar{v}_{i}=\sum_{i=1}^{n} \sum_{j=0}^{m} \theta_{j s} \rho^{-j} w_{i}{ }^{s-j}+E / \rho^{m+1},
$$

where

$$
\theta_{j s}=\sum_{r+t=j} \varphi_{r, s-1} \psi_{t} \text {. }
$$

Now $\sum_{i=1}^{n} w_{i}{ }^{s-j}$ is zero except when $j-s=k n$, where $k$ is integral, in which case it is $(-1)^{k} n$. Therefore, if we denote $k n+s$ by $z$, the double sum in (10) reduces to

$$
\sum_{k=0}^{I_{s}}(-1)^{k} n \theta_{z s} / \rho^{z}
$$

when $s=1,2, \cdots, n-1$, and to

$$
-n+\sum_{k=0}^{I_{s}}(-1)^{k} n \theta_{z s} / \rho^{z}
$$

when $s=n$. The symbol $I_{s}$ denotes the integral part of $(m-s) / n$. Substituting back into (9), we now get

$$
\begin{array}{r}
\sum_{k=0}^{I_{s}}(-1)^{k} n \theta_{i s} / \rho^{z}+\frac{1}{\rho^{m+1}}\left[\sum_{i=1}^{n}\left(w_{i}^{s}+E / \rho\right) \bar{E}_{i}+E\right]=0 \\
(s=1,2, \cdots, n) .
\end{array}
$$

Since these are identities in $\rho$, the coefficient of every power of $1 / \rho$ up to and including the $m$ th must vanish separately, giving the $m$ equations

$$
\begin{aligned}
\theta_{z s}=0, \quad \text { or } \quad \psi_{z} & =-\sum_{r+t=z}^{t<z} \varphi_{r, s-1} \psi_{t} \\
& \left(k=0,1, \cdots, I_{s} ; s=1,2, \cdots, n\right),
\end{aligned}
$$

and leaving the $n$ equations

$$
\sum_{i=1}^{n}\left(w_{i}{ }^{s}+E / \rho\right) \bar{E}_{i}+E=0 \quad(s=1,2, \cdots, n) .
$$

From equations (11) the $m$ functions $\psi_{j}$ may be determined in succession, and are readily shown by mathematical induction to have continuous derivatives of order $(m+n-j)$ in $(a, b)$. The functions represented by the letter $E$ in equations (12) can be expressed in terms of the $\varphi$ 's, the $\psi$ 's, and the $E_{i k}$ 's, 
and are therefore now to be regarded as known functions. When $|\rho|$ is sufficiently large the determinant of the coefficients in (12) is not zero, so the $\bar{E}_{i}$ 's can be uniquely determined. It is plain that for $\rho$ in $S_{k}$ they are analytic in $\rho$ and bounded as $\rho$ becomes infinite.

Bowdoin College, October, 1916.

\section{ON NOTATIONAL EQUIVALENCE.}

BY PROFESSOR EDWIN BIDWELL WILION.

IN reply to my query* to Dr. Poor "Why not make the work short?" he states $\dagger$ that brevity was not his aim, that one of his purposes was to exhibit the Burali-Forti and Marcolongo notation. I must accept that answer and admit my error in assuming that his only aim was to derive as directly as possible some transformations which are needed in certain studies in applied mathematics. It is, however, difficult for me to admit many of his other contentions. I have no desire to enter upon any polemic in regard to these matters, but it does seem that further explanation from Dr. Poor would be valuable to all who are interested in vectorial methods.

1. He states: That the use of words, such as grad, div, rot, is hampering seems to be a matter of opinion, since they may be used interchangeably with other symbols.

I hold that because two sets of symbols may be used as interchangeably as these and $\nabla$ is no criterion at all that one is not more hampering than another. For instance, 94 and XCIV are equivalent symbols, so are 8 and VIII, and also 752 and DCCLII. Yet for the arithmetical operation of multiplying eight and ninety-four the Arabic notation is far superior to the Roman (or Greek); indeed so marked is the superiority that one may well wonder how far mathematics would now be advanced had no better system than the Roman been devised.

May we not fairly maintain that notationally Arabic and Roman numerals are not interchangeable? Is it true that two notations in terms of which premises and conclusions may both be stated are for that reason interchangeable? To

* Wilson, this Bulletin, vol. 22, April, 1916, p. 336.

$\dagger$ Poor, this Bulletrin, vol. 22, July, 1916, p. 503. 